\title{
Estudio dieléctrico de cerámicas de textura y microestructura controladas con composiciones $\left(\mathrm{SrBi}_{2} \mathrm{Nb}_{2} \mathrm{O}_{9}\right)_{1-x}\left(\mathrm{Bi}_{3} \mathrm{TiNbO}_{9}\right)_{x}$
}

\author{
A. MOURE, R. JIMÉNEZ, C. ALEMANY, L. PARDO \\ Instituto de Ciencia de Materiales de Madrid, CSIC, Cantoblanco 28049, Madrid, España
}

\begin{abstract}
Se han preparado cerámicas de la solución sólida $\left(\mathrm{SrBi}_{2} \mathrm{Nb}_{2} \mathrm{O}_{9}\right)_{1-\mathrm{x}}\left(\mathrm{Bi}_{3} \mathrm{TiNbO}_{9}\right)_{\mathrm{x}}$ con $\mathrm{x}=0.35,0.65$ y 1.00 y estructura tipo Aurivillius obtenidas por sinterización natural y por prensado en caliente. Se parte de precursores amorfos obtenidos por activación mecanoquímica de una mezcla estequiométrica de óxidos y carbonatos, lo que permite utilizar temperaturas moderadas de procesado. Estos materiales son interesantes por su posible uso como piezoeléctricos de alta temperatura. La caracterización dieléctrica permite establecer a qué temperatura se encuentra la transición ferro-paraeléctrica que limita la temperatura de uso del material y como afectan las propiedades eléctricas, especialmente la conductividad d. c., a la polarizabilidad de las cerámicas, así como su relación con su microestructura y textura. En este trabajo se ha realizado el estudio dieléctrico de estos materiales en el intervalo de frecuencias de $100 \mathrm{~Hz}$ a $5 \mathrm{MHz}$ y en el rango de temperaturas desde $200{ }^{\circ} \mathrm{C}$

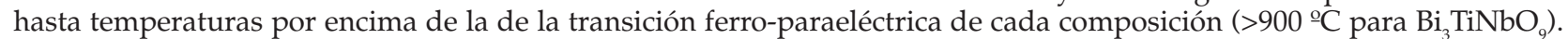

Palabras clave: Ferroeléctricos, Cerámicas, $\mathrm{SrBi}_{2} \mathrm{Nb}_{2} \mathrm{O}_{9}, \mathrm{Bi}_{3} \mathrm{TiNbO}_{9^{\prime}}$ Permitividad Dieléctrica.

Dielectric properties of ceramics of composition $\left(\mathrm{SrBi}_{2} \mathrm{Nb}_{2} \mathrm{O}_{9}\right)_{1-x}\left(\mathrm{Bi}_{3} \mathrm{TiNbO}_{9}\right)_{x}$ with controled texture and microstructure

Ceramics of composition $\left(\mathrm{SrBi}_{2} \mathrm{Nb}_{2} \mathrm{O}_{9}\right)_{1-\mathrm{x}}\left(\mathrm{Bi}_{3} \mathrm{TiNbO}_{9}\right)_{\mathrm{x}}$ with $\mathrm{x}=0.35,0.65$ and 1.00 and Aurivillius type structure have been prepared by natural sintering and hot pressing. Amorphous precursors were obtained by mechanochemical activation of stoichiometric mixtures of oxides and carbonates, which allows using moderate processing temperatures. These materials are interesting for their use as high temperature piezoelectrics. Dielectric characterisation allows to know the temperature at what takes place the ferro-paraelectric transition, which limits the working temperature of the material. It also gives information on how the electric properties, especially the d. c. conductivity, affect the polarizability of the ceramics. The properties relation with the microstructure and the texture is studied. In this work, dielectric studies of these materials have been made, in the frequency interval from $100 \mathrm{~Hz}$ to $5 \mathrm{MHz}$ and in the temperature range from $200{ }^{\circ} \mathrm{C}$ up to the ferroparaelectric transition temperatures $\left(>900{ }^{\circ} \mathrm{C}\right.$ for $\left.\mathrm{Bi}_{3} \mathrm{TiNbO}_{9}\right)$.

Keywords: Ferroelectrics, Ceramics, $\mathrm{SrBi}_{2} \mathrm{Nb}_{2} \mathrm{O}_{9}, \mathrm{Bi}_{3} \mathrm{TiNbO}_{9}$, Dielectric Permittivity.

\section{INTRODUCCIÓN}

Los compuestos con estructura tipo Aurivillius (1) tienen como fórmula general $\left[\mathrm{Bi}_{2} \mathrm{O}_{2}\right]\left[\mathrm{A}_{\mathrm{n}-1} \mathrm{~B}_{\mathrm{n}} \mathrm{O}_{3 \mathrm{n}+1}\right]$, constituida por $\mathrm{n}$ capas de pseudo-perovskita [ABO $]^{2-i n t e r c a l a d a s ~ c o n ~ c a p a s ~ d e ~}$ $\left[\mathrm{Bi}_{2} \mathrm{O}_{2}\right]^{2+}$. Muchos de estos compuestos son ferroeléctricos de alta temperatura de transición ferro-paraeléctrica que concentran en la actualidad gran interés como materiales piezoeléctricos para usos a alta temperatura (2). Además, su reducida fatiga, prolongada retención de polarización y bajas corrientes de fugas les hacen interesantes en la obtención de memorias ferroeléctricas no volátiles (FERAM) cuando se preparan en lámina delgada (3).

$\mathrm{Al}$ igual que en otras composiciones de similar estructura cristalina, es difícil obtener cerámicas densas del compuesto $\mathrm{Bi}_{3} \mathrm{TiNbO}_{9}$ y su solución sólida con $\mathrm{SrBi}_{2} \mathrm{Nb}_{2} \mathrm{O}_{9}(\mathrm{n}=2)$ cuando se preparan por síntesis de estado sólido debido a su hábito de crecimiento. Éste da lugar a partículas de morfología laminar (4), asemejándose a su estructura cristalina, de difícil compactación. Cuando se preparan por prensado en caliente las caras mayores de los granos se apilan en perpendicular a la presión aplicada, obteniéndose cerámicas muy densas pero anisótropas en sus propiedades (5). Una ruta alternativa se utiliza en este trabajo, en el que partiendo de precursores altamente reactivos se obtienen cerámicas de textura y microestructura controlada a temperaturas moderadas.

Las propiedades dieléctricas de estos materiales, y en especial su conductividad, son determinantes del rendimiento del proceso de polarización bajo la acción de campos eléctricos intensos y condicionan así mismo su respuesta piezoeléctrica a alta temperatura.

Se ha visto ya en trabajos previos y en cerámicas isótropas de BTN, preparadas a partir de precursores obtenidos por activación mecanoquímica, como el aumento de la porosidad y del tamaño de grano disminuye la permitividad dieléctrica (4). En cerámicas obtenidas por prensado en caliente se alcanzan densidades mayores que el $99 \%$ de la densidad teórica. Esa alta densificación es la responsable del aumento de la permitividad dieléctrica respecto de las muestras sinterizadas. Se ha observado (6) que conforme aumenta la temperatura de prensado disminuye el valor de $\varepsilon^{\prime}$. Como todas las cerámicas tienen densidades altas y muy parecidas, y son isótropas (por 
debajo de cierta temperatura de prensado) hay que buscar la diferencia en otros factores microestructurales de las cerámicas y que también influyen en la conductividad, como se verá más adelante.

La anisotropía de la muestra prensada en caliente a $1050^{\circ} \mathrm{C}$ durante 1 hora es consecuencia de la textura de la cerámica (7). A esta temperatura los granos se alinean con el eje c paralelo a la presión aplicada. En el que denominamos corte paralelo, con las caras mayores y plano paralelas de las muestras a medir paralelas a la presión aplicada, se mide la contribución a $\varepsilon^{\prime}$ del plano a-b, donde está el eje ferroeléctrico. De ahí que la muestra medida en esa dirección presente una $\varepsilon^{\prime}$ más alta, y la medida en la dirección perpendicular sea la que tenga el valor menor de todas las cerámicas medidas.

$\mathrm{Al}$ introducir en la composición de las cerámicas $\mathrm{SrBi}_{2} \mathrm{Nb}_{2} \mathrm{O}_{9}$ en solución sólida con $\mathrm{Bi}_{3} \mathrm{TiNbO}_{9}$ se rebaja la temperatura de transición ferro-paraeléctrica con respecto a la de ésta última, pudiéndose obtener así cerámicas ferroeléctricas con la transición de fase ferro-paraeléctrica dentro de un rango amplio de temperaturas. La relación entre la temperatura de transición y el contenido de $\mathrm{Bi}_{3} \mathrm{TiNbO}_{9}$ es lineal (2). El ensanchamiento en la transición producido en la solución sólida se ha visto en composiciones similares con $\mathrm{x}=0.25$, $0.50,0.75$ (2), y está asociado al carácter difuso de la transición ferro-paraeléctrica de la solución frente a los extremos de la misma.

Se han realizado en este trabajo medidas de impedancia de estos materiales hasta temperaturas por encima de la transición ferro-paraeléctrica. La relación de las características microestructurales de las cerámicas con las propiedades dieléctricas de estos materiales se discute en este estudio.

\section{MÉTODO EXPERIMENTAL}

Se han preparado cerámicas de composición $\left(\mathrm{SrBi}_{2} \mathrm{Nb}_{2} \mathrm{O}_{9}\right)_{1-\mathrm{x}}$ $\left(\mathrm{Bi}_{3} \mathrm{TiNbO}_{9}\right)_{\mathrm{x}}$, con $\mathrm{x}=0.35,0.65$ y 1.00 ha partir de una mezcla estequiométrica de $\mathrm{Bi}_{2} \mathrm{O}_{3}, \mathrm{Nb}_{2} \mathrm{O}_{5}, \mathrm{TiO}_{2}$ y $\mathrm{SrCO}_{3}$ de alta pureza. Esta mezcla es inicialmente homogeneizada en un mortero de ágata durante 3 minutos. Después, la mezcla se somete a molienda en un molino vibratorio (Fritsch Pulverisitte 0), con bola de $5 \mathrm{~cm}$ de diámetro, ambos de acero inoxidable, durante 336 horas. Así se obtiene una mezcla amorfa, de acuerdo al análisis por difracción de rayos X (8).

Este polvo precursor se prensa uniaxialmente a $300 \mathrm{Kg} \cdot \mathrm{cm}^{-2}$, conformando discos de unos $10 \mathrm{~mm}$ de diámetro y $2 \mathrm{~mm}$ de espesor. Estos discos de prensan isostáticamente a 2000 $\mathrm{Kg} \cdot \mathrm{cm}^{-2}$ y se sinterizan en horno eléctrico a temperaturas de 1000 a $1150^{\circ} \mathrm{C}$ durante 1 ó 2 horas. Otros discos se prensan en caliente en moldes de alúmina rodeados de polvo de alumina a temperaturas entre 700 y $1050 \stackrel{\circ}{\circ} \mathrm{C}$ y $\sim 200 \mathrm{Kg} \cdot \mathrm{cm}^{-2}$ durante 1 hora.

Las medidas de impedancia, entre $100 \mathrm{~Hz}-5 \mathrm{MHz}$ y en función de la temperatura se realizaron en un analizador HP4194A sobre cerámicas de caras paralelas, con electrodos de Pt sinterizado a $\sim 700^{\circ} \mathrm{C}$. El calentamiento se hizo a un ritmo de $2{ }^{\circ} \mathrm{C} / \mathrm{min}$, estabilizando la temperatura durante 1 minuto. Las medidas se realizaron tanto en subida como en bajada de la temperatura. A partir de estos datos experimentales se obtuvieron la permitividad compleja, $\varepsilon^{*}=\varepsilon^{\prime}-\mathrm{i} \varepsilon^{\prime \prime} \mathrm{y}$ por ajuste de los arcos de impedancia $Z^{\prime}-Z^{\prime \prime}$ la conductividad d. c., usando el programa EQUIVCRT (B. A. Boukamp. Ac Inmitance Data Analysis System Equivalent Circuit. Version 4.50. University of Twente. Twente.1999).

\section{RESULTADOS}

La caracterización microestructural mediante microscopía cuantitativa de cerámicas de $\mathrm{Bi}_{3} \mathrm{TiNbO}_{9}$ y el comportamiento de la parte real de la permitividad dieléctrica medida a 1 $\mathrm{MHz}$ frente a la temperatura (4) pone de manifiesto la dependencia de la misma tanto con la porosidad de la cerámica como con su tamaño de grano. Todas estas cerámicas sinterizadas son isótropas.

Se ha estudiado también cuantitativamente tanto la microestructura como la textura (7) de cerámicas de $\mathrm{Bi}_{3} \mathrm{TiNbO}_{9}$ obtenidas mediante prensado en caliente. Todas estas cerámicas tienen porosidades menores $(<1 \%)$ que las sinterizadas (del orden del 10\%). Dependiendo de la temperatura de tratamiento se pueden obtener cerámicas isótropas o texturadas con el eje c de la perovskita paralelo a la presión aplicada. Las cerámicas son isótropas preparadas a baja temperatura $\left(700^{\circ} \mathrm{C}\right.$ - $1000{ }^{\circ} \mathrm{C}$ ) debido a la ausencia del desarrollo de una morfología laminar, que se pone de manifiesto por estudios de TEM (9), característicos de los granos cerámicos en estos materiales. Estas cerámicas tienen valores más altos de $\varepsilon^{\prime}(6)$ que las cerámicas sinterizadas al aire ( $\varepsilon^{\prime} \sim 1100$ en el máximo), disminuyendo ésta conforme aumenta la temperatura de prensado. En la cerámica prensada a $1050{ }^{\circ} \mathrm{C}-1$ h se observa una gran diferencia en $\varepsilon^{\prime}$ en la medida de muestras con corte paralelo a la presión aplicada y el perpendicular a ésta.

En la figura 1 se muestra la variación con la temperatura de la permitividad dieléctrica para la solución sólida $\left(\mathrm{SrBi}_{2} \mathrm{Nb}_{2} \mathrm{O}_{9}\right)_{1-x}\left(\mathrm{Bi}_{3} \mathrm{TiNbO}_{9}\right)_{x}$ para $\mathrm{x}=0.35,0.65$ en cerámicas sinterizadas al aire. Hay una disminución de la temperatura de transición ferro-paraeléctrica conforme aumenta el contenido de $\mathrm{SrBi}_{2} \mathrm{Nb}_{2} \mathrm{O}_{9}$. Además se observa un ensanchamiento de la transición con respecto a la del $\mathrm{Bi}_{3} \mathrm{TiNbO}_{9}$. Cuando estas cerámicas se obtienen por prensado en caliente se produce un aumento de la permitividad dieléctrica medida en la dirección del corte paralelo a la presión aplicada. En la figura 2 se obser-

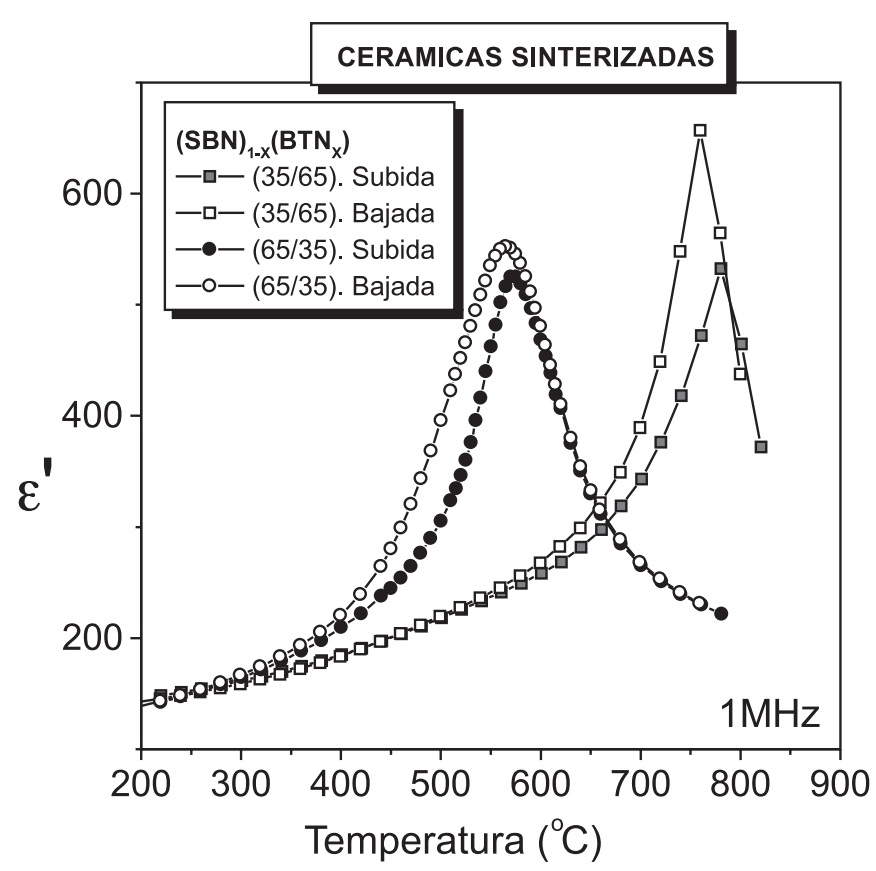

Figura 1. Parte real de la permitividad dieléctrica en función de la temperatura para cerámicas sinterizadas a $1150{ }^{\circ} \mathrm{C}-2 \mathrm{~h}$ de $\left(\mathrm{SrBi}_{2} \mathrm{Nb}_{2} \mathrm{O}_{9}\right)_{1-x}\left(\mathrm{Bi}_{3} \mathrm{TiNbO}_{9}\right)_{x}$. 


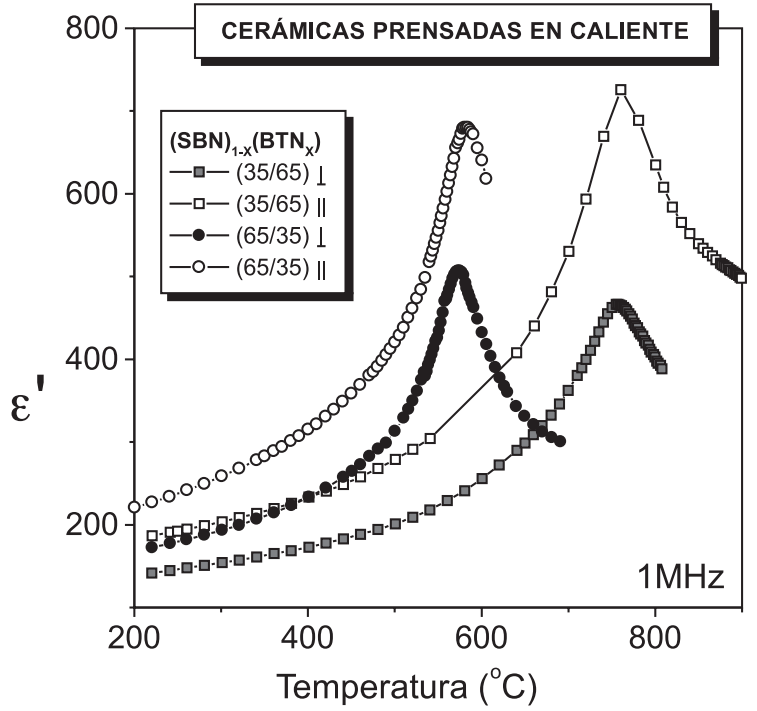

Figura 2. Parte real de la permitividad dieléctrica en función de la temperatura para cerámicas prensadas en caliente a $1050{ }^{\circ} \mathrm{C}-1 \mathrm{~h}$ de $\left(\mathrm{SrBi}_{2} \mathrm{Nb}_{2} \mathrm{O}_{9}\right)_{1-\mathrm{x}}\left(\mathrm{Bi}_{3} \mathrm{TiNbO}_{9}\right)_{\mathrm{x}}$.

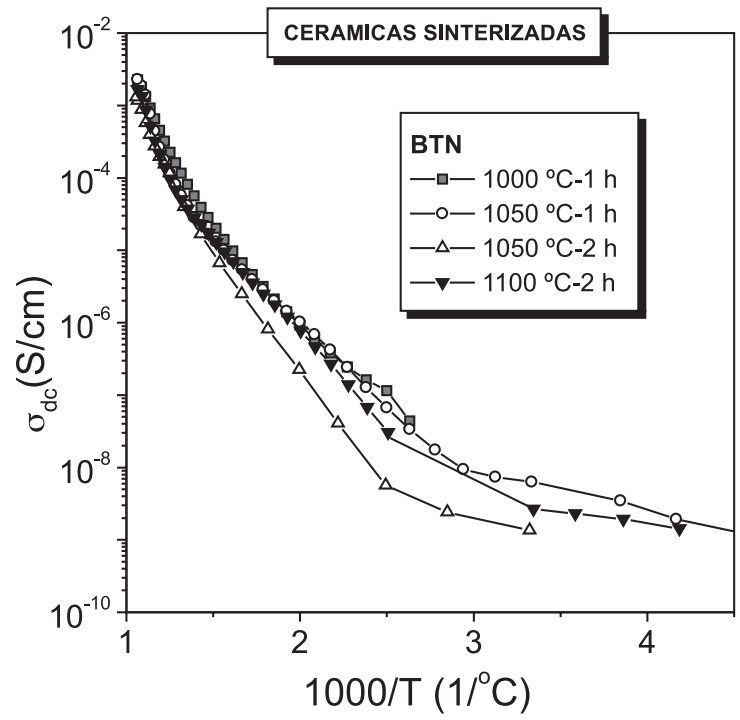

Figura 4. Conductividad d. c en función de la temperatura para cerámicas prensadas en caliente de $\mathrm{Bi}_{3} \mathrm{TiNbO}_{9}$

va asimismo la diferencia entre las dos direcciones de corte para ambas composiciones.

La conductividad d. c. en subida de las cerámicas de $\mathrm{Bi}_{3} \mathrm{TiNbO}_{9}$ sinterizado al aire se puede ver en la figura 3. En general la conductividad disminuye al aumentar la temperatura de tratamiento, excepto para la cerámica preparada a $1100{ }^{\circ} \mathrm{C}$ durante 2 horas. Aunque no muy definidos a altas temperaturas, se pueden distinguir 3 tramos en la forma de las curvas. Las cerámicas prensadas en caliente (figura 4) son algo más conductoras que las sinterizadas, sobre todo cuando son prensadas a temperaturas más bajas $\left(700^{\circ} \mathrm{C}\right)$. En la muestra prensada a $1050{ }^{\circ} \mathrm{C}-1 \mathrm{~h}$ se aprecia una diferencia de conductividad en los dos cortes, siendo el paralelo a la presión aplicada el más conductor.

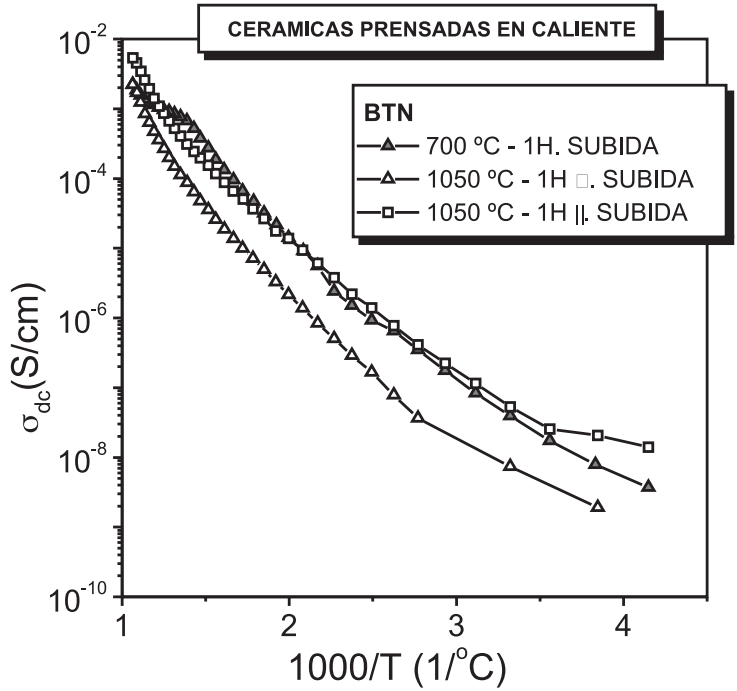

Figura 3. Conductividad d. c. en función de la temperatura para cerámicas sinterizadas de $\mathrm{Bi}_{3} \mathrm{TiNbO}_{9}$

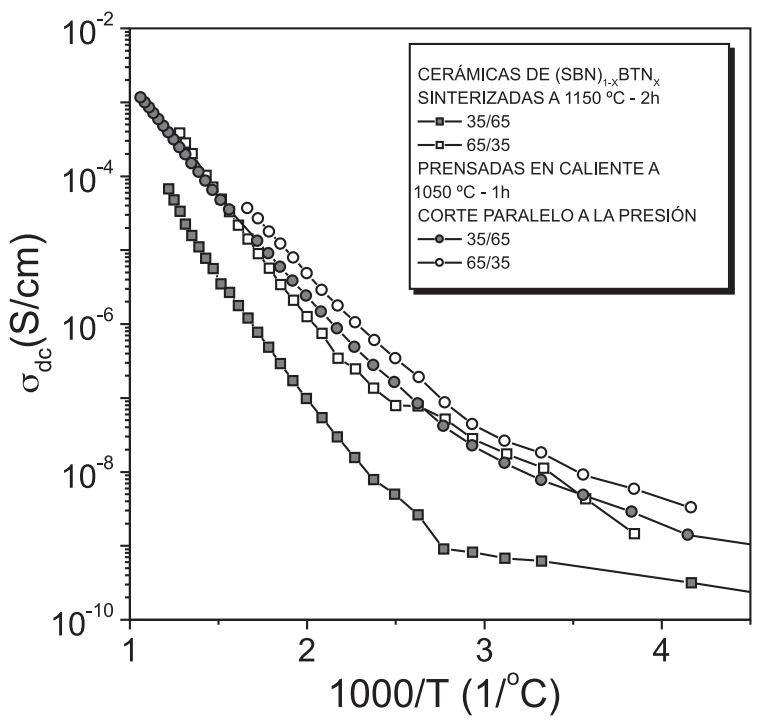

Figura 5. Conductividad d. c en función de la temperatura para cerámicas de $\left(\mathrm{SrBi}_{2} \mathrm{Nb}_{2} \mathrm{O}_{9}\right)_{1-x}\left(\mathrm{Bi}_{3} \mathrm{TiNbO}_{9}\right)_{x}$

La figura 5 muestra la conductividad de la solución sólida frente a la temperatura. La cerámica con $\mathrm{x}=0.65$ sinterizada a $1150{ }^{\circ} \mathrm{C}-2 \mathrm{~h}$ es menos conductora que la sinterizada en las mismas condiciones con $\mathrm{x}=0.35 \mathrm{y}$ que las prensadas en caliente a $1050^{\circ} \mathrm{C}-1 \mathrm{~h}$.

\section{DISCUSIÓN}

La medida de conductividad d. c. se ha hecho por ajuste de los arcos de impedancia Z'- Z'”. Según la temperatura de medida se pueden ver 1, 2 y hasta 3 semicírculos. En otros estudios (10) se asocia cada semicírculo a la conducción por volumen, por borde de grano y por la intercara cerámica-electrodo. La conductividad total del material se toma como la 
suma en serie de cada elemento. La impedancia de la muestra está dominada por el elemento de mayor R.

A temperaturas bajas son distinguibles 2 semicírculos. En composiciones de $\mathrm{Bi}_{3} \mathrm{Ti}_{4} \mathrm{O}_{12}$ dopadas con $\mathrm{Nb}$ se propone que es el borde de grano el que domina la conducción a bajas temperaturas (11). Se puede asociar en nuestro caso uno de los semicírculos a la conducción por borde de grano y en serie con este el de volumen. Esto explicaría que las cerámicas con grano más pequeño $(<1 \mu \mathrm{m},(5))$, y por tanto más densidad de borde de grano por volumen, fuesen más conductoras.

$\mathrm{Al}$ aumentar la temperatura sólo aparece un semicírculo. La contribución de borde de grano se hace menor y es la de volumen la que domina. En los dos tipos de cerámicas (prensadas y sinterizadas) la conductividad en este rango de temperaturas disminuye al aumentar la temperatura de sinterización. Este comportamiento debe tener causas microestructurales relacionadas con el tamaño y forma de los granos.

Para estudiar el efecto de la forma de los granos, que en estos materiales está relacionado con el tamaño, hemos empleado el modelo desarrollado por C. Pecharromán (12) de campo efectivo con percolación y aplicado por R. Jiménez (13) a propiedades dieléctricas, en el que se supone una distribución al azar de partículas con forma de esferoide y distinta conductividad en el eje c y el plano a-b perpendicular a éste. No se han considerado en el modelo la influencia de las fronteras de grano ni de las paredes de dominio. Variando el factor de llenado (porosidad) de la muestra la conductividad disminuye, tal como se aprecia en las cerámicas. Independientemente de la densidad, se observa que al aumentar la relación plano $(\mathrm{a}=\mathrm{b}) / \mathrm{c}$ la conductividad de volumen disminuye (figura 6). Al aumentar la temperatura de tratamiento se favorece esa relación axial (4), por lo que debe disminuir la conductividad tal y como ocurre en este caso.

Sin embargo, la diferencia en conductividad prevista por el modelo no explica totalmente el obtenido experimentalmente. A bajas temperaturas debe haber una influencia importante del borde de grano. Además, el modelo no considera la influencia de las paredes de dominios en la conductividad. El hecho de que la conductividad de las cerámicas sea convergente al pasar la temperatura de transición a la fase paraeléctrica, donde no hay dominios ferroeléctricos puede indicar que éstos tienen un papel importante en la conductividad d. c. Se ha propuesto (14) que en estos materiales se produce un anclaje de carga en las paredes de dominio de $90^{\circ}$. En las cerámicas con grano muy pequeño no se favorece la creación de dominios de este tipo. En este caso, esa carga está más libre y produce un aumento en la conductividad. Al llegar a la fase paraeléctrica, donde han desaparecido los dominios, la conductividad se iguala aproximadamente para cada forma y tamaño de grano, pareciéndose más a lo predicho por el modelo. Al enfriar, la carga vuelve a anclarse aunque a un ritmo menor que antes. Esto produce una histéresis térmica y hace que las conductividades medidas en bajada se hagan más parecidas. Sin embargo, no es comparable la bajada en cerámicas prensadas a $700{ }^{\circ} \mathrm{C}$, ya que al superarse en la medida esta temperatura se produce una recristalización, y consiguiente cambio de microestructura, con lo que las propiedades dieléctricas difieren de la del calentamiento.

De nuevo, la diferencia entre los dos cortes de la cerámica prensada a $1050{ }^{\circ} \mathrm{C}-1$ hora hay que buscarlos en la textura, ya que en el corte paralelo se mide en el plano a-b, mucho más conductor que el c. Eso provoca las diferencias en la conductividad en cada corte.

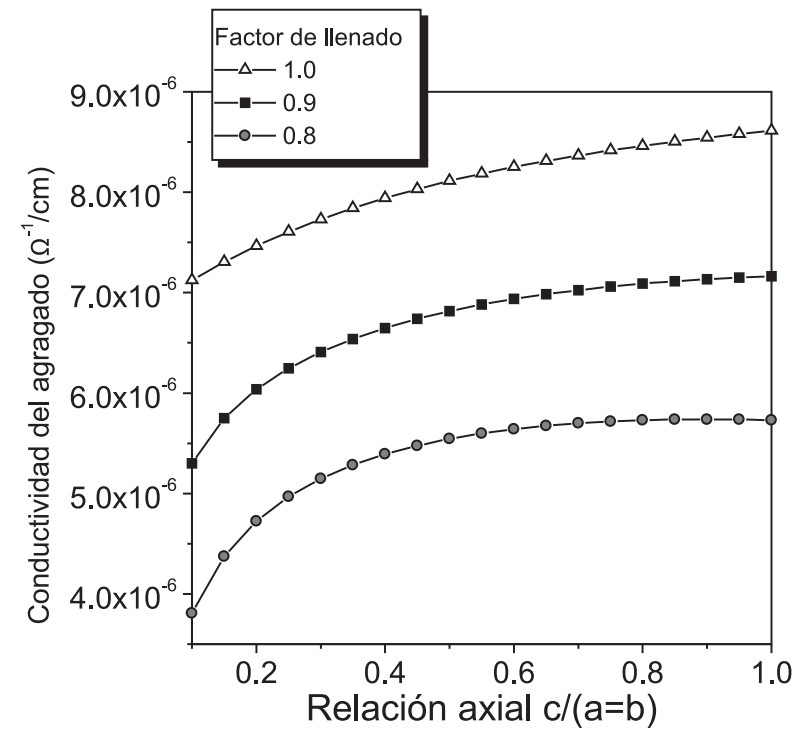

Figura 6. Conductividad en función de la relación axial calculada por el modelo de C. Pecharromán y aplicado por R. Jiménez a propiedades dieléctricas para una distribución al azar de partículas con forma de esferoide y distinta conductividad en el eje c y el plano a-b perpendicular a éste

En cuanto a la forma de la curva de conductividad frente a temperatura, en todas las cerámicas se distinguen 3 tramos, excepto en la prensada a $700 \stackrel{\circ}{\circ} \mathrm{C}$ durante una hora. En ésta aparecen 4 tramos. La meseta a temperaturas cercanas a $700^{\circ} \mathrm{C}$ debe estar producida por la recristalización que se está produciendo. La energía térmica aplicada produce más una reorganización de cargas que un aumento de la conductividad eléctrica. Por las limitaciones experimentales no es posible ajustar los circuitos equivalentes a bajas temperaturas. En las que sí se ha podido hacer (sinterizada a $1050^{\circ} \mathrm{C} 1$ hora) se observa en esa zona una conductividad casi independiente de la temperatura, como ya se ha visto en cerámicas de composiciones similares de $\mathrm{SrBi}_{2} \mathrm{Ta}_{2} \mathrm{O}_{9}$ y $\mathrm{SrBi}_{2} \mathrm{Nb}_{2} \mathrm{O}_{9}$ (10).

En las soluciones sólidas se observa como el incremento de $\mathrm{SrBi}_{2} \mathrm{Nb}_{2} \mathrm{O}_{9}$ hace las cerámicas más conductoras. Esto es debido a que al disminuir la cantidad de $\mathrm{Bi}_{3} \mathrm{TiNbO}_{9}$ en la solución el rango de temperaturas de medida está mucho más cerca de la transición (donde hay un aumento de la conductividad).

De nuevo conducen más las cerámicas cortadas en paralelo a la presión aplicada (medidas en el plano más conductor) que cuando se prensan en caliente. La textura desarrollada por las cerámicas no es tan fuerte como la de $\mathrm{Bi}_{3} \mathrm{TiNbO}_{9}$. La anisotropía es más evidente en la permitividad que en la conductividad, debido a la transición de fase ferro-paraeléctrica. Cerca de la transición la diferencia en el pico de $\varepsilon^{\prime}$ es más acusado al medirlo en cada plano. Sin embargo no hay evidencia de que al acercarse a la transición haya cambios en el mecanismo de conducción entre los 2 planos. Así, la diferencia de conducción en los 2 cortes en cerámicas no muy texturadas no es muy grande. Además, las muestras sinterizadas (más porosas) tienen una conductividad menor que el corte perpendicular a la presión. En este caso hay una contribución de un número algo mayor de granos conduciendo a través del eje c, pero es más importante el efecto de la porosidad, que limita los caminos de conducción y la cantidad de fase conductora de la cerámica. 


\section{CONCLUSIONES}

A partir de precursores amorfos, se han preparado cerámicas de textura y microestructura controlada mediante sinterización natural y prensado en caliente. El estudio dieléctrico realizado en cerámicas cuya microestructura ha sido previamente caracterizada indica que:

- las cerámicas de $\mathrm{Bi}_{3} \mathrm{TiNbO}_{9}$ tienen una permitividad dieléctrica menor que las prensadas en caliente por su mayor porosidad y son isótropas eléctricamente;

- la conductividad d. c. en muestras isótropas se hace mayor cuando disminuye la porosidad de las cerámicas, y disminuye al aumentar el tamaño de grano y aumentar la relación $(\mathrm{a}=\mathrm{b}) / \mathrm{c}$;

- en cerámicas prensadas en caliente, la textura desarrollada al prensarlas a $1050^{\circ} \mathrm{C}$ durante 1 hora provoca una anisotropía eléctrica, que se pone de manifiesto en los valores de permitividad y conductividad medidas en los cortes perpendicular o paralelo a la presión, teniendo éste último valores más altos en ambos casos.

En cerámicas de composición $\left(\mathrm{SrBi}_{2} \mathrm{Nb}_{2} \mathrm{O}_{9}\right)_{1-x}\left(\mathrm{Bi}_{3} \mathrm{TiNbO}_{9}\right)_{x}$ se observa un aumento en la conductividad cuando se disminuye la cantidad de $\left(\mathrm{Bi}_{3} \mathrm{TiNbO}_{9}\right)$ en la solución, debido a una disminución de la temperatura de transición ferro-paraeléctrica.

\section{AGRADECIMIENTOS}

Este trabajo ha sido cofinanciado por los proyectos MAT97-0711 de la CICYT, 07N/0061/1998 de la CAM y LEAF de la UE (contrato G5RD-CT2001-00431). Los autores agradecen a la Dra. A. Castro la preparación de los precursores activados mecanoquímicamente

\section{BIBLIOGRAFÍA}

1. B. Aurivillius. Mixed bismuth oxides with layer lattices. Arkiv Kemi, 1, 463480 (1949)

2. B. Jiménez, L. Pardo, A. Castro, P. Millán, R. Jiménez, M. Elaatmani y M. Oualla. Influence of the preparation on the microstructure and ferroelectricity of the (SBN) $)_{1-x}(\mathrm{BTN})_{x}$. Ferroelectrics, 241, 279-286, (2000).

3. C. A. Paz de Araujo, J. D. Cuchiaro, L. D. M Millan, M. C. Scott y J. F. Scott. Fatigue-free ferroelectric capacitors with platinum electrodes. Nature, 374, 627, (1995).

4. L. Pardo, A. Moure, A. Castro, P. Millán, C. Alemany y B. Jiménez. Microestructura y piezoelectricidad de cerámicas de $\mathrm{Bi}_{3} \mathrm{TiNbO}_{9}$ obtenidas a partir de precursores activados mecanoquímicamente. Bol. Soc. Esp. Cerám. Vidrio, 38, 6, 563-567, (1999).

5. P. Durán-Martín. Propiedades ferroeléctricas de materiales cerámicos con estructura tipo Aurivillius de composiciones basadas en $\mathrm{SrBi}_{2} \mathrm{Nb}_{2} \mathrm{O}_{9}$. Tesisi Doctoral UAM (1997).

6. L. Pardo, A. Moure, J. Ricote, A. Castro y P. Millán. Piezoceramics from mechanochemically activated precursors. Ferroelectrics 2000 UK Workshop Proceedings. 97-104. ISBN 1-86125-135-1.

7. A. Moure. L. Pardo, C. Alemany, P. Millán, A. Castro. Piezoelectric ceramics based on $\mathrm{Bi}_{3} \mathrm{TiNbO}_{9}$ from mechanochemically activated precursors. J. Eur. Ceram. Soc (en prensa).

8. A. Castro, P. Millán, L. Pardo y B. Jiménez. Synthesis and sintering improvement of Aurivillius type structure ferroelectric ceramics by mechanochemical activation. J. Mater. Chem., 9, 1313-1317, (1999).

9. J. Ricote, L. Pardo, A. Moure, A. Castro, P. Millán y D. Chateigner. "Microcharacterisation of grain oriented ceramics based on BTN from mechanochemical activated precursors". J. Eur. Ceram. Soc. (En prensa).

10. T.C. Chen, C.L. Thio, S. B. Desu. Impedance spectroscopy of $\mathrm{SrBi}_{2} \mathrm{Ta}_{2} \mathrm{O}_{9}$ and $\mathrm{SrBi}_{2} \mathrm{Nb}_{2} \mathrm{O}_{9}$ ceramic correlation with fatigue behavior. J. Mater. Res., 12 (10), 2628-2637, (1997).

11. P. Durán-Martín, C. Voisard, D. Damjanovic y N. Setter. Study and control of the conductivity of $\mathrm{Nb}$-doped $\mathrm{Bi}_{4} \mathrm{Ti}_{3} \mathrm{O}_{12}$ for high temperature piezoelectric applications. Bol. Soc. Esp. Cerám. Vidrio, 38, 6, 582-586, (1999).

12. C. Pecharromán y J.E. Iglesias. Effective dielectric properties of packed mixtures of insulator particles. Phys. Rev. B. , 49, 11. 7137-7147. (1994)

13. R. Jiménez. Application of the Percolation Effective Medium Theory (PEMT) to solid electrolyte pressed powder pellets: II. Electrically anisotropic solid electrolyte particles. Solid State Ionics, 92, 225-241, (1996).

14. B. Jiménez, A. Castro, L. Pardo, P. Millán y R. Jiménez. Electric and ferro-piezoelectric properties of $(\mathrm{SBN})_{1-x}(\mathrm{BTN})_{x}$ ceramics obtained from amorphous precursors. J. Phys. Chem. Solids, (2000) (en prensa).

Recibido: 31.05 .01

Aceptado: 10.12 .01 